03,09

\title{
Зависимость светоэкситонного взаимодействия от ширины квантовой ямы во внешнем однородном электрическом поле
}

\author{
() Д.К. Логинов ${ }^{1}$, А.В. Донец ${ }^{2}$ \\ ${ }^{1}$ Лаборатория оптики спина им. И.Н. Уральцева Санкт-Петербургского государственного университета, \\ Санкт-Петербург, Россия \\ ${ }^{2}$ Санкт-Петербургский государственный университет, \\ Санкт-Петербург, Россия \\ E-mail: loginov999@gmail.com
}

Поступила в редакцию 14 декабря 2020 г.

В окончательной релакции 14 декабря 2020 г.

Принята к публикации 16 декабря 2020 г.

Теоретически анализируется зависимость светоэкситонного взаимодействия от размеров квантовой ямы во внешнем электрическом поле. Показано, что в достаточно сильном поле увеличение ширины ямы приводит к ослаблению светоэкситонного взаимодействия по сравнению с более узкими ямами. Произведены численные расчеты продольно-поперечного расщепления экситона для широкого диапазона электрических полей и значений ширины квантовой ямы. Анализ полученных данных позволил получить выражения, описывающие зависимости светоэкситонного взаимодействия как от величины приложенного электрического поля, так и от ширины ямы.

Ключевые слова: экситон, электрическое поле, квантовая яма, светоэкситонное взаимодействие.

DOI: $10.21883 /$ FTT.2021.04.50709.238

\section{1. Введение}

Спектроскопия широких квантовых ям (КЯ) является наиболее удобным методом исследования состояний движущегося экситона [1-13]. Широкими называются ямы, толщина слоя которых на порядок превосходит экситонный боровский радиус $[4,6,12]$. В таких широких ямах происходит размерное квантование движения экситона как целого, что приводит к появлению дискретных уровней энергии, проявляющихся в оптических спектрах.

Эти исследования получили свое дальнейшее развитие в работах, посвященных изучению структур с широкими КЯ во внешних полях. Внешние воздействия приводят к новым эффектам, которые можно наблюдать с помощью оптической спектроскопии. Например, поле одноосного сжатия, приложенное к широкой КЯ, индуцирует эффект сближения масс экситонов тяжелой и легкой дырок и приводит к инверсии фазы спектральных осцилляций экситонных особенностей в оптических спектрах [14,15]. Магнитное поле, в зависимости от ориентации относительно оси квантования, приводит либо к эффективному увеличению массы экситона, либо к зависимости $g$-фактора экситона от волнового вектора его движения [16-24].

Важным случаем внешнего воздействия является однородное электрическое поле. К настоящему моменту большая часть работ, обсуждающих эффекты электрического поля, посвящена исследованию объемных полупроводников, см., например, [25-35]. В этих работах обсуждается, в частности, штарковский сдвиг энергии экситона в различных полупроводниковых материалах.
Штарковский сдвиг изучался также в узких КЯ. Было показано, что пространственное ограничение экситона уменьшает величину эффекта Штарка [36-39]. Следует отметить, что в объемных полупроводниках свет не может возбуждать состояния экситона с импульсом, отличающимся от импульса фотона, который для большей части спектральной области мал по сравнению с импульсом экситона и может считаться нулевым. В то же время в узких КЯ центр масс экситона не может двигаться вдоль оси квантования из-за того, что ширина слоя ямы меньше размера экситона. Поэтому в работах [25-39] фактически рассматривался случай, в котором внешнее электрическое поле воздействует на неподвижный экситон.

Влияние электрического поля на состояния движущегося экситона можно исследовать в оптических спектрах широких КЯ. В одной из первых работ такого рода была теоретически проанализирована электрооптическая функция $P$-экситона в режиме осцилляций ФранцаКелдыша в плоскопараллельной пластинке [40]. В работах $[41,42]$ рассмотрен индуцированный электрическим полем эффект инверсии фазы спектральных осцилляций особенностей отражения, связанных с уровнями размерного квантования экситона в КЯ. Авторами работ $[42,43]$ исследовано влияние электрического поля на светоэкситонное взаимодействие в широкой КЯ. Показано, что электрическое поле должно приводить к уменьшению амплитуды экситонных осцилляций отражения в спектрах широкой ямы.

Настоящая работа развивает теоретические исследования, выполненные в работах $[42,43]$, в которых, в частности, исследована зависимость светоэкситонного 
взаимодействия от электрического поля только для одной ширины КЯ GaAs/AlGaAs. В настоящей работе показано, что эта зависимость меняется с изменением ширины ямы. Изменение светоэкситонного взаимодействия выражается в размерной зависимости величины продольно-поперечного расщепления экситона в электрическом поле. В рамках анализа этой зависимости рассчитаны значения продольно-поперечного расщепления для различных значений толщины широких КЯ. Получены математические выражения, описывающие эту зависимость для широких ям.

\section{2. Теория}

Рассматривается экситон в кристалле GaAs, кристаллическая решетка которого имеет симметрию цинковой обманки. Считаем, что экситон двигается вдоль оси $z$, направление которой совпадает с кристаллографической осью [001]. Вдоль этого же направления действует внешнее однородное электрическое поле $F$. Оси $x$ и $y$ выбраны вдоль осей четвертого порядка [100] и [010].

Гамильтониан экситона является суммой гамильтониана свободного электрона, гамильтониана Латтинжера для дырки [44], их кулоновского взаимодействия и энергии, обусловленной действием внешнего электрического поля.

Заметим, что в кубических кристаллах типа GaAs состояния экситонов тяжелых и легких дырок смешиваются членами гофрировки дырочного гамильтониана и потому, в общем случае, не могут рассматриваться независимо [45]. Однако в нашей работе мы рассматриваем два типа состояний, для которых в первом приближении можно пренебречь эффектами смешивания экситонов легкой и тяжелой дырки. Первый из них - состояния с большим волновым вектором, $K \gg 1 / a_{B}$, где $a_{B}-$ боровский радиус экситона в объемном кристалле [45]. Второй тип состояний, который важен для дальнейшего рассмотрения, связан с основным оптическим переходом экситона, который в спектрах дает наибольший по интенсивности вклад. Для состояния основного оптического перехода с хорошей точностью можно считать $K=0$. Такое приближение позволяет исключить из рассмотрения эффекты смешивания экситонов с тяжелой и легкой дырками, связанные с движением экситона. Чтобы еще сильнее упростить анализ, будем считать приведенную массу электрона и дырки в экситоне сферически-симметричной. Это приведет к погрешности в энергии связи экситона, которая, как показывает анализ, не превышает $10 \%$ и слабо влияет на обсуждаемый эффект. Такое упрощение позволяет исключить из рассмотрения члены в экситонном гамильтониане, связанные с гофрировкой валентной зоны. В этом случае можно ограничиться рассмотрением только состояний экситонов с тяжелой дыркой, которые наиболее ярко проявляются в оптических спектрах [9].

Для дальнейшего анализа удобно перейти от координат свободных электрона $\left(x_{e}, y_{e}, z_{e}\right)$ и дырки $\left(x_{h}, y_{h}, z_{h}\right)$ к координатам центра масс экситона вдоль оси $z$, $Z=\left(z_{e} m_{e}+m_{h} z_{h}\right) / M$, и координатам относительного движения электрона и дырки, $x=x_{e}-x_{h}, y=y_{e}-y_{h}$, $z=z_{e}-z_{h}$. Здесь $M=m_{e}+m_{h}-$ масса экситона, $m_{e}$ и $m_{h}$ - эффективные массы электрона и дырки, соответственно. После перехода к новым координатам гамильтониан экситона можно представить в виде

$$
\hat{H}=E_{g}+\hat{H}_{K}+\hat{H}_{p}
$$

Здесь $E_{g}$ - ширина запрещенной зоны. Второй оператор описывает движение экситона по кристаллу как целого и имеет вид

$$
\hat{H}_{K}=\frac{\hbar^{2} K^{2}}{2 M} .
$$

Третье слагаемое в гамильтониане (1) описывает относительное движение электрона и дырки. Оно имеет вид

$$
\hat{H}_{P}=-\frac{\hat{p}^{2}}{2 \mu}-\frac{e^{2}}{\varepsilon_{0} r}+e F z .
$$

Здесь $\hat{p}^{2}=-\hbar^{2} \partial_{x}^{2}-\hbar^{2} \partial_{y}^{2}-\hbar^{2} \partial_{z}^{2}-$ оператор квадрата импульса относительного движения электрона и дырки, $e$ - заряд электрона, $\varepsilon_{0}-$ фоновая диэлектрическая проницаемость, $r$ - расстояние между электроном и дыркой. Величина $\mu=m_{e} m_{h}\left(m_{e}+m_{h}\right)-$ приведенная мacca.

Для вычисления волновой функции и энергии основного состояния экситона в однородном электрическом поле используем стандартный метод разделения переменных в параболических координатах $[46,47]$. Для этого перейдем от декартовых к параболическим координатам, согласно формулам

$$
\xi=r+z, \quad \eta=r-z, \quad \varphi=\arctan (x / y) .
$$

В параболических координатах выражение для волновой функции принимает вид

$$
\phi=A f_{v}(\eta) g_{v^{\prime}}(\xi) e^{ \pm i m \varphi}, v^{\prime}=v+\mu e^{2} /\left(\varepsilon_{0} \hbar^{2}\right),
$$

где $A$ - нормировочный коэффициент, а функции $f_{v}(\eta)$ и $g_{v^{\prime}}(\zeta)$ являются решениями двух одномерных уравнений

$$
\begin{aligned}
& \frac{1}{\eta} \frac{d}{d \eta}\left(\eta \frac{d f_{v}(\eta)}{d \eta}\right)+\left(-\frac{m}{4 \eta^{2}}-\frac{v}{\eta}+\frac{\mu e F \eta}{4 \hbar^{2}}\right) f_{v}(\eta)=0 \\
& \frac{1}{\xi} \frac{d}{d \xi}\left(\eta \frac{d g_{v}(\xi)}{d \xi}\right)+\left(-\frac{m}{4 \xi^{2}}+\frac{v}{\eta}+\frac{\mu e F \zeta}{4 \hbar^{2}}\right) g_{v}(\xi)=0 .
\end{aligned}
$$

В этих уравнениях $R$ - энергия относительного движения электрона и дырки, а $v$ - параметр разделения переменных. Со светом взаимодействуют только $s$-состояния экситона, для которых проекция орбитального момента на выделенную ось $m=0$. Поэтому в уравнении (6) слагаемые, содержащие $m$, можно опустить. Далее, эти дифференциальные уравнения могут быть решены с помощью численных методов с граничными условиями, 

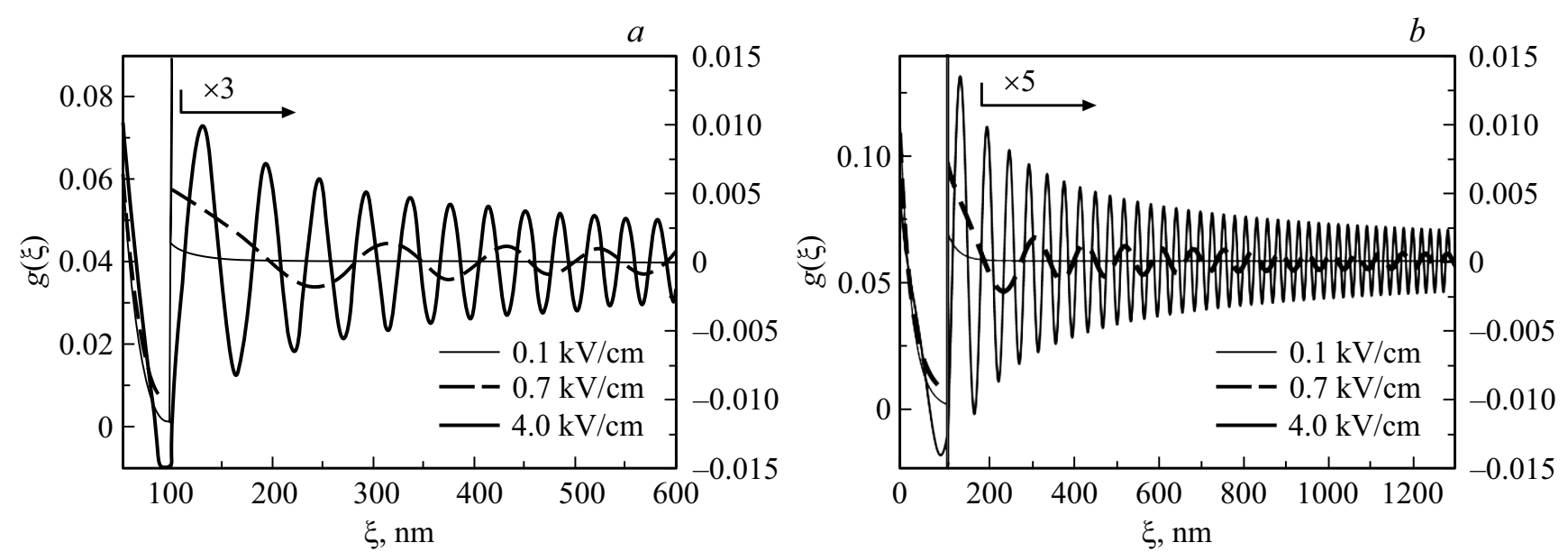

Рис. 1. Графики осциллирующей части волновой функции $g_{v}(\zeta)$, в электрических полях $F=0.1,0.7$ и $4 \mathrm{kV} / \mathrm{cm}, \mathrm{paccчитанные} \mathrm{для}$ КЯ толщиной $L=300 \mathrm{~nm}(a)$ и $L=600 \mathrm{~nm}(b)$. Левая шкала приведена для начального участка функций, $\xi<100 \mathrm{~nm}$.

которые накладывают на них гетерограницы КЯ. Детали численного решения системы уравнений (6) приведены в работе [43]. Здесь мы приведем только полученные результаты.

При достаточно больших значениях поля решениями уравнений (6) являются две волновые функции, одна из которых, $f_{v}(\eta)$, быстро затухает с ростом координаты $\eta$, а вторая, $g_{v}(\xi)$, является осциллирующей функцией координаты $\xi$. Примеры осциллирующих волновых функций $g_{v}(\zeta)$, рассчитанных для КЯ толщиной $L=300 \mathrm{~nm}$ и $L=600 \mathrm{~nm}$, приведены на рис. 1. Вид затухающей функции $f_{v}(\eta)$ при всех приложенных электрических полях аналогичен виду функции $g_{v}(\xi)$ в малом поле $F=0.1 \mathrm{kV} / \mathrm{cm}$ и поэтому не представлен на рисунке. Заметим, что чем больше ширина КЯ, тем больше частота осциллирующей функции и тем меньше ее амплитуда в любой точке $\xi$ по сравнению с $g_{v}(\xi)$ в более узкой КЯ.

Полученные функции $f_{v}(\eta)$ и $g_{v}(\xi)$ позволяют найти искомую волновую функцию относительного движения в соответствии с (5). Нормировочный коэффициент $A$ определяется условием

$$
A^{2} \iint f_{V}^{2}(\eta) g_{v}^{2}(\zeta) \frac{1}{4}(\eta+\zeta) d \eta d \zeta d \varphi=1,
$$

в котором интегрирование проводится по объему КЯ единичной площади. Для вычисления функций $f_{v}(\eta)$ и $g_{v}(\xi)$ нами были использованы следующие материальные параметры GaAs: $\varepsilon_{0}=12.56$ [48], $m_{e}=0.067 m_{0}$ [48], $m_{h}=0.45 m_{0}$ [49]. Заметим, что при вычислении $f_{v}(\eta)$ и $g_{v}(\xi)$ было использовано приближение, в рамках которого волновая функция (5) обращается в ноль не на плоских интерфейсах КЯ, а на поверхности некоторого параболоида $[42,43]$. С одной стороны, такие граничные условия упрощают наши вычисления в параболических координатах [43]. С другой стороны, в работе [42] показано, что полученные в рамках такого приближения результаты хорошо согласуются с расчетом, использующим точные граничные условия.
Волновая функция (5) входит в выражение для продольно-поперечного расщепления, определяющего величину светоэкситонного взаимодействия, следующим образом [1]:

$$
\hbar \omega_{L T}=\left(\frac{2 e P_{1}}{E_{g}}\right)^{2} \frac{\pi}{\varepsilon_{0}}|\phi(0)|^{2} .
$$

Здесь $\phi(0)$ - волновая функция относительного движения при $r=0$; материальная константа $P_{1}=\hbar p_{c v} / m_{0}$, где $p_{c v}-$ межзонный матричный элемент импульса. Для GaAs величина $P_{1}=10.3 \cdot 10^{-5} \mathrm{meV} \cdot \mathrm{cm}[50]$.

Светоэкситонное взаимодействие зависит от приложенного электрического поля, которое влияет на волновую функцию относительного движения электрона и дырки в экситоне. Используя рассчитанные выше волновые функции, мы можем вычислить по формуле (8) продольно-поперечные расщепления экситона для различных значений толщины КЯ.

\section{3. Результаты и обсуждение}

На рис. 2 представлены результаты расчетов величины $\hbar \omega_{L T}$ для толщин КЯ $L$ от 200 до $2500 \mathrm{~nm}$. Анализ показывает, что диапазон рассматриваемых значений приложенного поля можно условно разделить на три области значений. В каждой из этих областей зависимость продольно-поперечного расщепления от размера ямы имеет свои особенности.

В относительно небольших полях, $F \leq 0.4 \mathrm{kV} / \mathrm{cm}$, для всех рассмотренных значений $L$ зависимость $\hbar \omega_{L T}(F)$ выглядит одинаково. Это связано с тем, что в таких относительно слабых полях экситон практически не ионизирован.

Электрон и дырка при этом практически не покидают кулоновскую яму их взаимного притяжения, и состояние относительного движения электрона и дырки близко к состоянию водородоподобного экситона. Эта ситуация 

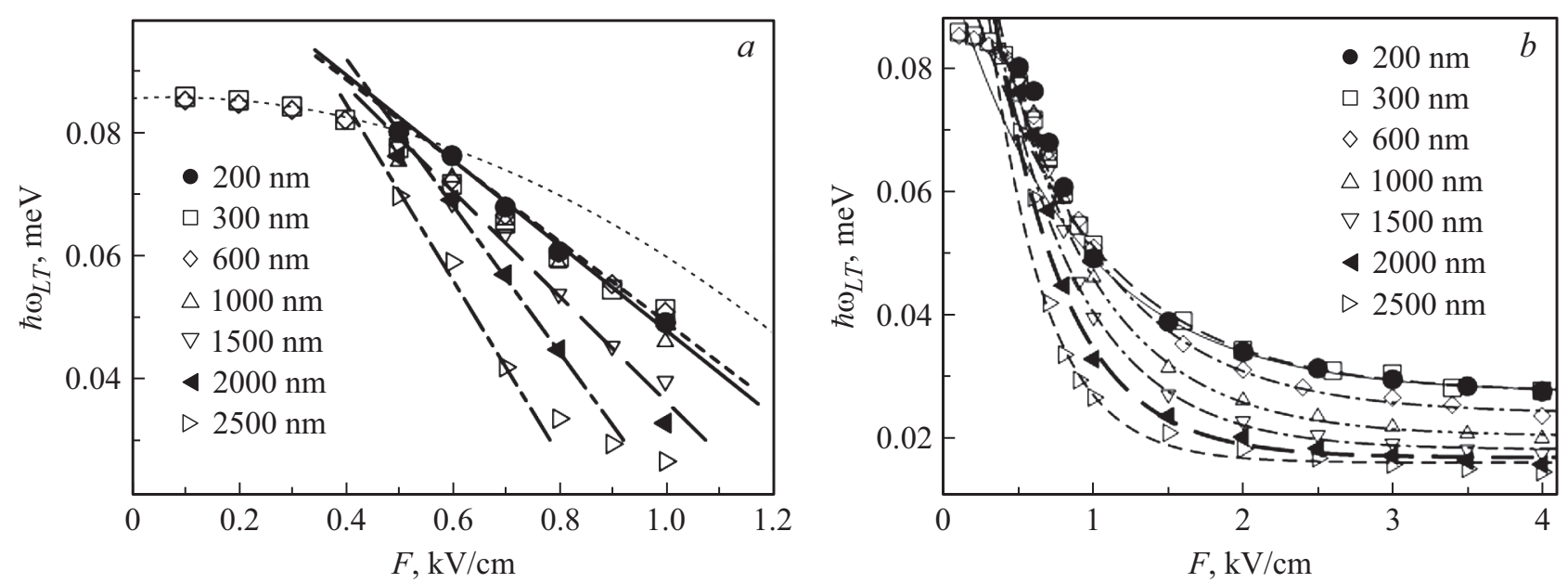

Рис. 2. Зависимости продольно-поперечного расщепления экситона в КЯ толщиной $L$ от 200 до $2500 \mathrm{~nm}$ от величины внешнего электрического поля. Точки - результат численного расчета. Рисунок $(a)$ демонстрирует в увеличенном масштабе зависимости $\hbar \omega_{L T}(F)$ в полях $F \geq 1.2 \mathrm{kV} / \mathrm{cm}$. Пунктирная кривая описывается выражением (9), которое хорошо подгоняет расчетные данные для $F \geq 0.4 \mathrm{kV} / \mathrm{cm}$. Прямые линии описываются выражениями (10) и (11), которые применимы для величины приложенного поля $0.4 \mathrm{kV} / \mathrm{cm}<F<0.8-1 \mathrm{kV} / \mathrm{cm}$ в зависимости от ширины слоя GaAs. Кривые на рисунке $(b)$ определяются выражениями (12) и (13) в тексте статьи.

может быть проиллюстрирована кривыми $g_{v}(\xi)$, рассчитанными для поля $F=0.1 \mathrm{kV} / \mathrm{cm}$ (см. рис. 1 ). Как можно видеть, у функции $g_{v}(\xi)$ в таком малом поле практически отсутствуют осцилляции, и за пределами кулоновской ямы ее амплитуду можно считать равной нулю. В этом случае, относительное движение электрона и дырки не „чувствует“ гетерограниц достаточно широкой КЯ, как это было показано в работе [12]. Поэтому в широких КЯ и в полях $F \leq 0.4 \mathrm{kV} / \mathrm{cm}$ волновая функция, а как следствие, и продольно-поперечное расщепление (8) не меняются при изменении ширины ямы. Учитывая это обстоятельство, мы привели на рис. 2, a зависимость $\hbar \omega_{L T}(F)$ только для КЯ с ширинами $L=300$ и $600 \mathrm{~nm}$ для значений приложенного поля $F \leq 10.4 \mathrm{kV} / \mathrm{cm}$. Для остальных кривых в этой области малых значений поля зависимости продольнопоперечного расщепления выглядят идентично. Все они могут быть аппроксимированы аналитической кривой вида:

$$
\hbar \omega_{L T}(F)=\hbar \omega_{L T}(0)\left(1+0.051 F-0.35 F^{2}\right) .
$$

где $\hbar \omega_{L T}(0)=0.087 \mathrm{meV}$ - продольно-поперечное расщепление в отсутствие поля. Это значение хорошо согласуется с результатами экспериментального исследования продольно-поперечного расщепления экситона в $\mathrm{GaAs}$ методом рамановского рассеяния: $\hbar \omega_{L T}(0)=0.085 \mathrm{meV}$, см., например, [51].

В более сильном электрическом поле зависимость $\hbar \omega_{L T}(F)$ можно аппроксимировать линейной функцией:

$$
\hbar \omega_{L T}^{(L)}(F)=a_{2}(L) F+b_{2}(L) .
$$

Здесь коэффициенты $a_{2}(L)$ и $b_{2}(L)$ зависят от ширины КЯ. При этом верхняя граница этой области полей также зависит от $L$. Так, для значений ширины ямы $L<1500 \mathrm{~nm}$ область, в которой продольно-поперечное расщепление хорошо аппроксимируется выражением (10), соответствует $0.4 \mathrm{kV} / \mathrm{cm}<F<1 \mathrm{kV} / \mathrm{cm}$. При этом, как демонстрирует рис. 2, $a$, для $L<1000 \mathrm{~nm}$ коэффициенты в выражении (10) совпадают в пределах видимой погрешности, а для $L=1500 \mathrm{~nm}$ они очень близки к значениям для $L=1000 \mathrm{~nm}$.

Для КЯ шириной $L=2000 \mathrm{~nm}$ зависимость (10) справедлива при $0.4 \mathrm{kV} / \mathrm{cm}<F<0.9 \mathrm{kV} / \mathrm{cm}$. Для ямы шириной $L=2500 \mathrm{~nm}$ зависимость (10) справедлива в диапазоне полей $0.4 \mathrm{kV} / \mathrm{cm}<F<0.8 \mathrm{kV} / \mathrm{cm}$. Мы построили графики зависимостей коэффициентов $a_{2}$ и $b_{2}$ от ширины ямы, которые изображены на рис. 3. Кривые, проведенные через расчетные точки, - аппроксимации параболами, которые верны для ширины КЯ $L \geq 600 \mathrm{~nm}$. Они имеют вид:

$$
\begin{aligned}
& a_{2}(L)=1.71 \cdot 10^{-8} \cdot L^{2}+1.063 \cdot 10^{-5} \cdot L-0.0586, \\
& b_{2}(L)=3.5 \cdot 10^{-9} \cdot L^{2}+7.23 \cdot 10^{-6} \cdot L+0.101
\end{aligned}
$$

В этих выражениях ширина ямы $L$ выражена в нанометрах. Для ширины ямы $L \leq 600 \mathrm{~nm}$ эти коэффициенты постоянны и равны $a_{2}=0.0578 \mathrm{meV} \cdot \mathrm{cm} / \mathrm{kV}$ и $b_{2}=0.1061 \mathrm{meV}$.

Наличие зависимости от $L$ в выражениях (10) и (11) связано с тем, что в полях $0.4 \mathrm{kV} / \mathrm{cm}<F<0.8 \div 1 \mathrm{kV} / \mathrm{cm}$ электрон и дырка покидают кулоновскую яму их взаимного притяжения, и становится значительной вероятность обнаружить их вдали от начала координат их относительного движения. Математически это обстоятельство выражается в том, что у волновой функции (5) появляются осциллирующие хвосты (см. рис. 1), на которые 


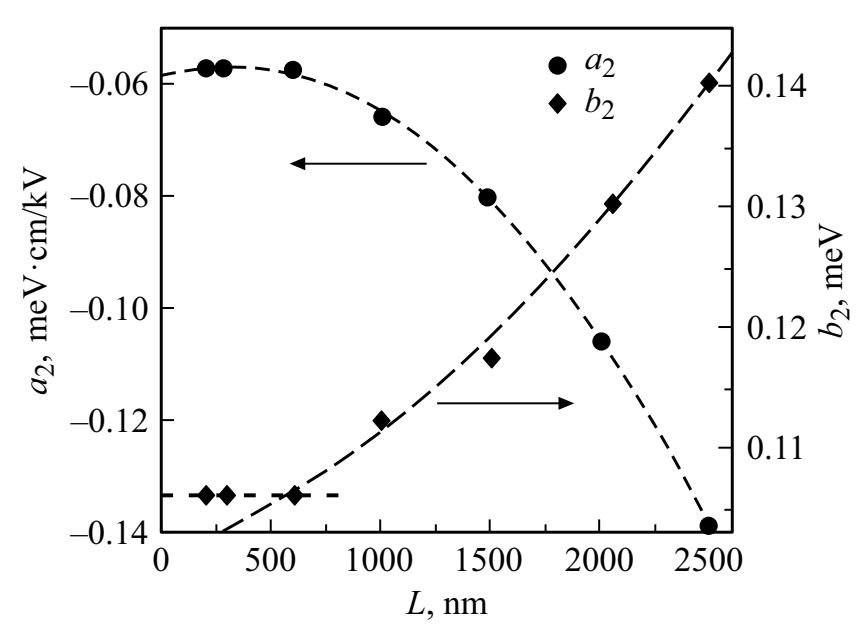

Pис. 3. Зависимости от ширины ямы $L$ коэффициентов $a_{2}$ и $b_{2}$ в выражении (10). Точки - результат численного расчета. Кривые - аппроксимации формулами (11).

накладываются нулевые граничные условия на интерфейсах КЯ. При этом длина хвостов определяется шириной КЯ. Вследствие требований нормировки, удлинение хвостов волновой функции приводит к уменьшению ее значений в начале координат $\phi(0)$, и, как следствие, к уменышению продольно-поперечного расщепления, согласно выражению (8). Следовательно, увеличение $L$ должно приводить к уменьшению $\hbar \omega_{L T}(F)$.

Наконец, в диапазоне самых сильных электрических полей, $F>1 \mathrm{kV} / \mathrm{cm}$, зависимость продольно-поперечного расщепления имеет вид

$$
\hbar \omega_{L T}^{(L)}(F)=a_{3}(L) \exp \left(-b_{3}(L) F\right)+c_{3}(L) .
$$

Здесь коэффициенты $a_{3}(L), b_{3}(L)$ и $c_{3}(L)$ зависят от ширины КЯ. Полученные нами теоретические значения величины (12) представлены в виде графиков на рис. 4, a и $b$. Расчетные точки могут быть аппроксимированы кривыми, представленными на том же рисунке. Используя функции, которым соответствуют эти кривые, можем описать зависимость параметров в выражении (12) от $L$ следующим выражениями:

$$
\begin{aligned}
& a_{3}(L)=0.0113 \cdot L^{1 / 3}+0.042 \\
& b_{3}(L)=2.333 \cdot 10^{-7} \cdot L^{2}+1.22 \\
& c_{3}(L)=3.716 \cdot 10^{-9} \cdot L^{2}-1.252 \cdot 10^{-5} \cdot L+0.031
\end{aligned}
$$

Таким образом, в наиболее сильных электрических полях зависимость от величины $F$ становится более слабой, как это демонстрирует рис. 2, $b$. Это связано с тем, что вероятность обнаружить электрон и дырку за пределами кулоновской ямы по порядку величины равна вероятности обнаружить их внутри этой ямы. При этом действие приложенного поля, стремящегося ионизировать экситон, компенсируется ограничением движения электрона и дырки потенциальными барьерами КЯ. Поэтому увеличение электрического поля слабо меняет величину продольно-поперечного расщепления. На языке математического описания это значит, что, как и в случае промежуточного поля $(0.4 \mathrm{kV} / \mathrm{cm}<F<0.8 \div 1 \mathrm{kV} / \mathrm{cm})$, наличие относительного движения электрона и дырки вне кулоновской ямы потенциала их притяжения приводит к тому, что величина $\phi(0)$ уменьшается с увеличением ширины КЯ. Однако в сильных полях из-за ограничения интерфейсами КЯ изменение амплитуды осциллирующих хвостов волновой функции несущественно. Как следствие, значения $\phi(0)$ и продольнопоперечное расщепление (8) изменяются тоже довольно слабо.

Таким образом, мы получили выражения, описывающие зависимость продольно-поперечного расщепления в широких КЯ и планарных слоях как от электрического поля, так и от ширины ямы или слоя. Заметим также, что из полученных результатов следует, что для гетероструктур на основе GaAs/AlGaAs наиболее сильная размерная зависимость продольно-поперечного расщепления должна наблюдаться при значениях ширины КЯ более $1000 \mathrm{~nm}$.

Заметим, что в идеализированном случае экситона в КЯ электрон и дырка не могут разлететься друг от друга на бесконечность даже в сильном поле, если оно направлено поперек плоскости ямы. Выражаясь квазиклассическим языком, из-за того, что электрон и дырка отражаются от границ КЯ, они периодически возвращаются в кулоновскую яму их взаимного притяжения. Поэтому ионизация экситона не происходит ни при каких значениях электрического поля, и экситонные состояния стационарны.

Если в рамках мысленного эксперимента устремить ширину КЯ к бесконечности, то полученные в нашей модели решения будут также соответствовать стационарным состояниям. Поскольку осциллирующая часть $g_{\nu^{\prime}}(\xi)$ волновой функции (5) является незатухающей, а также из-за требования нормировки, при условии $L \rightarrow \infty$ амплитуда волновой функции $\phi$ будет стремиться к нулю в каждой точке пространства. Как следствие, такое стационарное состояние экситона не должно давать вклад в оптические спектры. Однако, как известно, например, из работ [28-30], спектральные особенности, связанные с экситонами, наблюдаются в оптических спектрах объемных полупроводников в электрическом поле. Это связано с тем, что в объемном кристалле состояние экситона становится нестационарным, поскольку электрон и дырка, после того как покинут кулоновскую яму их взаимного притяжения, не могут более вернуться обратно. Экситон при этом имеет конечное время жизни, которое определяется туннелированием носителей заряда через кулоновский барьер в электрическом поле. Кроме того, в реальной структуре сушествует рассеяние электрона и дырки при их движении вне кулоновской ямы, которое также ограничивает время жизни экситона. Похожая ситуация имеет место для двумерных экситонов в электрическом поле, направленном в плоскости 

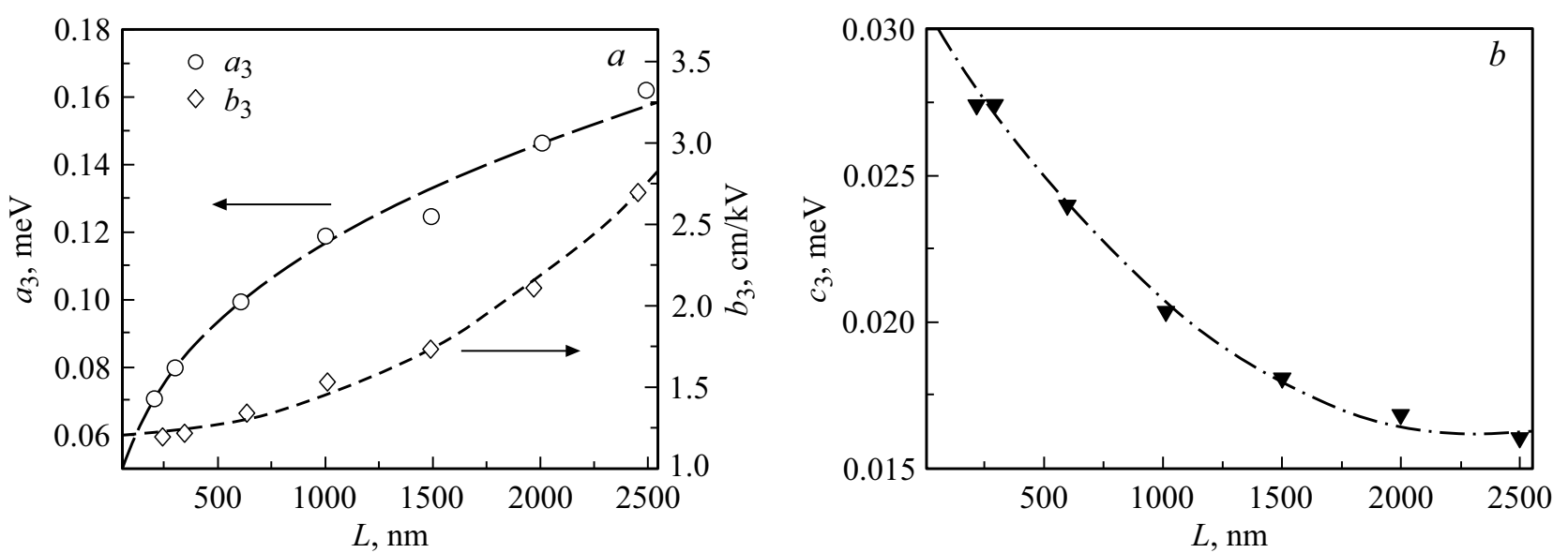

Рис. 4. Зависимости от ширины ямы $L$ коэффициентов $a_{3}, b_{3}(a)$ и $c_{3}(b)$ в выражении (12). Точки - результат численного расчета. Кривые - аппроксимации формулами (13).

КЯ, см., например, работы [52,53]. Поэтому, чтобы осуществить переход от рассмотренного нами случая КЯ к объемному полупроводнику, следует установить, при каких условиях экситонное состояние становится нестационарным.

Чтобы это сделать, заметим, что рассеяние на примесях или фононах ограничивает время жизни экситона не только в объемном полупроводнике, но и в слое КЯ. Испытав рассеяние за пределами кулоновской ямы, электрон и дырка могут не вернуться обратно, а разлететься на бесконечное расстояние в плоскости КЯ. Экситон при этом распадается как в объемном кристалле. Подобные процессы можно охарактеризовать величиной среднего свободного пробега, $l$, как это сделано, например, в работе [30]. При выполнении условия

$$
l \ll L
$$

можно считать, что относительное движение не чувствует гетерограниц КЯ, и экситонные состояния в электрическом поле следует рассматривать как нестационарные.

Величина среднего свободного пробега зависит от качества слоя КЯ и температуры. В простейшей модели можно считать, что величина $l$ постоянна для любого значения приложенного поля [30]. Определение этой величины является нетривиальной задачей и выходит за рамки настоящей работы. Отметим только, что в работе [54] было показано, что в достаточно чистых образцах GaAs при температуре жидкого гелия величина $l$ может достигать $3 \mu \mathrm{m}$. При таком значении среднего свободного пробега электрона условие (14) не выполняется для всех рассмотренных нами значений $L$, и полученные выше результаты остаются в силе. Однако если качество гетероструктур низкое либо измерения проводятся при высокой температуре, величина $l$ может стать малой и условие (14) будет выполняться для большинства рассмотренных значений ширины КЯ. При этом продольно-поперечное расщепление и экситонный вклад в оптические спектры будут вести себя в электрическом поле в соответствие с теориями, описывающими экситон в объемном кристалле.

Экспериментальное наблюдение описанного выше эффекта возможно в спектрах отражения широких КЯ с высоким структурным совершенством. Продольнопоперечное расщепление экситона определяет амплитуду наблюдаемых спектральных особенностей. Для экспериментального наблюдения зависимости светоэкситонного взаимодействия от размера КЯ можно сравнивать экспериментальные спектры гетероструктур с ямами различной ширины, измеренные при одинаковой величине приложенного поля. Обычно в широких КЯ удается наблюдать особенности размерного квантования движения экситона и доминирующую по амплитуде особенность основного оптического перехода [4-13]. Последняя связана с точкой антипересечения светоподобной и экситоноподобной дисперсионных поляритонных веток. Экспериментальные исследования экситонных оптических спектров широких КЯ, выполненные ранее, например, в работах [4-9], демонстрируют, что амплитуда осцилляций, связанных с состояниями размерного квантования движения экситона, уменьшается с шириной ямы. В частности, согласно работе [9], при $L>800 \mathrm{~nm}$ амплитуда этих состояний даже в высококачественных гетероструктурах близка к амплитуде шумов. Это делает невозможным наблюдение состояний размерного квантования экситона в тех КЯ, в которых зависимость продольно- поперечного расщепления от их ширины проявляется наиболее ярко $(L>1000 \mathrm{~nm})$.

В то же время в оптических спектрах КЯ, ширина которых превышает $800 \mathrm{~nm}$, будет наблюдаться спектральная особенность, соответствующая основному экситонному переходу. Энергия этого состояния экситона не зависит от размеров КЯ. Однако, как следует из полученных выше результатов, интенсивность спектральной особенности, определяемая продольно-поперечным расщеплением экситона, должна сильно зависеть от ширины ямы при приложении достаточно большого электрического поля. Следовательно, описанное выше явление 
можно экспериментально наблюдать по зависимости интенсивности пика основного экситонного перехода от ширины КЯ. Наиболее ярко этот эффект будет выражен в структурах с КЯ шириной более $1000 \mathrm{~nm}$.

\section{4. Заключение}

В работе выполнены численные расчеты продольнопоперечного расщепления экситона во внешнем однородном продольном электрическом поле в КЯ $\mathrm{GaAs} / \mathrm{AlGaAs}$ шириной от 200 до $2500 \mathrm{~nm}$. Показано, что эффект уменьшения светоэкситонного взаимодействия в электрическом поле зависит от ширины КЯ. Чем больше эта ширина, тем слабее свето-экситонное взаимодействие. Получены аналитические выражения, описывающие эту размерную зависимость для КЯ GaAs/AlGaAs при произвольном значении ширины ямы. Показано, что существуют три области значений величины приложенного поля, в которых зависимость от размера КЯ имеет разный характер и описывается различными выражениями.

\section{Финансирование работы}

Работа финансировалась Российским фондом фундаментальных исследований (РФФИ, грант № 19-02-00576). Авторы благодарят СПбГУ за финансовую поддержку в рамках гранта № 73031758 .

\section{Конфликт интересов}

Авторы заявляют, что у них нет конфликта интересов.

\section{Список литературы}

[1] E.L. Ivchenko. Optical Spectroscopy of Semiconductor Nanostructures. Alpha Science, Harrow (2005).

[2] A.V. Kavokin, J.J. Baumberg, G. Malpuech, F.P. Laussy. Microcavities. Oxford University, N.Y. (2007).

[3] C.F. Klingshirn. Semiconductor Optics. 4th ed. Springer, Berlin (2012).

[4] В.А. Киселев, И.В. Макаренко, Б.С. Разбирин, И.Н. Уральцев. ФТТ 20, 8, 1348 (1977).

[5] A. Tredicucci, Y. Chen, F. Bassani, J. Massies, C. Deparis, G. Neu. Phys. Rev. B 47, 10348 (1993).

[6] N. Tomassini, A. D’Andrea, R. Del Sole, H. Tuffigo-Ulmer, R.T. Cox. Phys. Rev. B 51, 5005 (1995).

[7] E. Ubyivovk, Yu.K. Dolgikh, Yu.P. Efimov, S.A. Eliseev, I.Ya. Gerlovin, I.V. Ignatiev, V.V. Petrov, V.V. Ovsyankin. J. Lumin. 102, 751 (2003).

[8] С.А. Марков, Р.П. Сейсян, В.А. Кособукин. ФТП 38, 230 (2004).

[9] Д.К. Логинов, Е.В. Убыйвовк, Ю.П. Ефимов, В.В. Петров, С.А. Елисеев, Ю.К. Долгих, И.В. Игнатьев, В.П. Кочерешко, А.В. Селькин. ФТТ 48, 1979 (2006) [Phys. Solid State 48, 2100 (2006)].

[10] Е.В. Убыйвовк, Д.К. Логинов, И.Я. Герловин, Ю.К. Долгих, Ю.П. Ефимов, С.А. Елисеев, В.В. Петров, О.Ф. Вывенко, А.А. Ситникова, Д.А. Кириленко. ФТТ 51, 1818 (2009).
[11] A.V. Trifonov, S.N. Korotan, A.S. Kurdyubov, I.Ya. Gerlovin, I.V. Ignatiev, Yu.P. Efimov, S.A. Eliseev, V.V. Petrov, Yu.K. Dolgikh, V.V. Ovsyankin, A.V. Kavokin. Phys. Rev. B 91, 115307 (2015).

[12] E.S. Khramtsov, P.A. Belov, P.S. Grigoryev, I.V. Ignatiev, S.Yu. Verbin, Yu.P. Efimov, S.A. Eliseev, V.A. Lovtcius, V.V. Petrov, S.L. Yakovlev. J. Appl. Phys. 119, 184301 (2016).

[13] E.S. Khramtsov, P.S. Grigoryev, D.K. Loginov, I.V. Ignatiev, Yu.P. Efimov, S.A. Eliseev, P.Yu. Shapochkin, E.L. Ivchenko, M. Bayer. Phys. Rev. B 99, 035431 (2019).

[14] D.K. Loginov, A.V. Trifonov, I.V. Ignatiev. Phys. Rev. B 90, 075306 (2014).

[15] D.K. Loginov, P.S. Grigoryev, Yu.P. Efimov, S.A. Eliseev, V.A. Lovtcius, V.V. Petrov, E.V. Ubyivovk, I.V. Ignatiev. Phys. Status Solidi B 253, 1537 (2016).

[16] J.J. Davies, D. Wolverson, V.P. Kochereshko, A.V. Platonov, R.T. Cox, J. Cibert, H. Mariette, C. Bodin, C. Gourgon, E.V. Ubylvovk, Y.P. Efimov, S.A. Eliseev. Phys. Rev. Lett. 97, 187403 ( 2006).

[17] A. Litvinov, V.P. Kochereshko, L. Besombes, H. Mariette, D. Loginov, J.J. Davies, L.C. Smith, D. Wolverson. Acta Phys. Pol. A 112, 2, 161 (2007).

[18] D. Loginov, V.P. Kochereshko, A. Litvinov, L. Besombes, H. Mariette, J.J. Davies, L.C. Smith, D. Wolverson. Acta Phys. Pol. A 112, 381 (2007).

[19] L.C. Smith, J.J. Davies, D. Wolverson, S. Crampin, R.T. Cox, J. Cibert, H. Mariette, V.P. Kochereshko, M. Wiater, G. Karczewski, T. Wojtowicz. Phys. Rev. B 78, 085204 (2008).

[20] Д.К. Логинов, В.П. Кочерешко, А.В. Платонов, J.J. Davies, D. Wolverson, L.C. Smith, R.T. Cox, J. Cibert, H. Mariette. ФTT 51, 1555 (2009).

[21] J.J. Davies, L.C. Smith, D. Wolverson, A. Gust, C. Kruse, D. Hommel, V.P. Kochereshko. Phys. Rev. B 81, 085208 (2010).

[22] L.C. Smith, J.J. Davies, D. Wolverson, H. Boukari, H. Mariette, V.P. Kochereshko, R.T. Phillips. Phys. Rev. B 83, 155206 (2011).

[23] P.S. Grigoryev, V.G. Davydov, S.A. Eliseev, Yu.P. Efimov, V.A. Lovtcius, P.Yu. Shapochkin, I.V. Ignatiev, M. Bayer. Phys. Rev. B 96, 155404 (2017).

[24] S.Yu. Bodnar, P.S. Grigoryev, D.K. Loginov, V.G. Davydov, Yu.P. Efimov, S.A. Eliseev, V.A. Lovtcius, E.V. Ubyivovk, V.Yu. Mikhailovskii, I.V. Ignatiev. Phys. Rev. B 95, 195311 (2017).

[25] Е.Ф. Гросc. УФН 76, 433 (1962). [E.F. Gross. Usp. Fiz. Nauk 76, 433 (1962)].

[26] A. Frova, P. Handler, F.A. Germano, D.E. Aspnes. Phys. Rev. 145, 575 (1966).

[27] D.F. Blossey. Phys. Rev. B 2, 3976 (1970).

[28] I.A. Merkulov, V.I. Perel. Phys. Lett. A 45, 83 (1973).

[29] И.А. Меркулов. ЖЭТФ 66, 2314 (1974).

[30] A. Jaeger, G. Weiser. Phys. Rev. B 58, 10674 (1998).

[31] A.T. Winzer, G. Gobsch, R. Goldhahn, D. Fuhrmann, A. Hangleiter, A. Dadgar, A. Krost. Phys. Rev. B 74, 125207 (2006).

[32] S. Lenk, E. Runge. J. Phys. Conf. Ser. 210, 012047 (2010).

[33] S. Zielinska-Raczynska, D. Ziemkiewicz, G. Czajkowski. Phys. Rev. B 94, 045205 (2016).

[34] J. Heckotter, M. Freitag, D. Frohlich, M. Abmann, M. Bayer, M.A. Semina, M.M. Glazov. Phys. Rev. B 95, 035210 (2017). 
[35] J. Heckotter, M. Freitag, D. Frohlich, M. Abmann, M. Bayer, M.A. Semina, M.M. Glazov. Phys. Rev. B 98, 035150 (2018).

[36] F. Bassani, G. Czajkowski, M. Dressler, L. Silvestri. Phys. Status Solidi B 178, 51 (2000).

[37] Y.-H. Kuo, Y.K. Lee, Y. Ge, S. Ren, J.E. Roth, T.I. Kamins, D.A.B. Miller, J.S. Harris. Nature 437, 1334 (2005).

[38] E. Kasapoglua, H. Saria, M. Bursala, I. Sökmen. Physica E 16, 237 (2003).

[39] L. Zhang, H.-Z. Duan, X.-F. Wang. Phys. Lett. A 373, 2969 (2009).

[40] S. Zielinska-Raczynska, D. Ziemkiewicz, G. Czajkowski. Phys. Rev. B 97, 165205 (2018).

[41] D.K. Loginov, A.V. Donets. Semicond. 52, 562 (2018).

[42] D.K. Loginov, P.A. Belov, V.G. Davydov, I.Ya. Gerlovin, I.V. Ignatiev, A.V. Kavokin, Y. Masumoto. Phys. Rev. Res. 2, 033510 (2020).

[43] Д.К. Логинов, А.В. Донец. ФТТ 62, 208 (2020).

[44] E.L. Ivchenko, G. Pikus. Superlattices and Other Microstructures, Springer-Verlag, Berlin (1995).

[45] E.O. Kane. Phys. Rev. B 11, 3850 (1975).

[46] Л.Д. Ландау, Е.М. Лифшиц. Квантовая механика. Нерелятивистская теория. М., Наука (1989). Гл. ХХ. С. 344.

[47] D.F. Blossey. Phys. Rev. B 3, 1382 (1971).

[48] G.E. Stillman, D.M. Larsen, C.M. Wolfe, R.C. Brandt. Solid State Commun. 9, 2245 (1971)

[49] S. Adachi. GaAs and Related Materials. World Scientific Publishing, Singapore (1994).

[50] Г.Е. Пикус, В.А. Марущак, А.Н. Титков. ФТП 22, 185 (1988).

[51] R.G. Ulbrich, C. Weisbuch. Phys. Rev. Lett. 38, 865 (1977).

[52] S. Haastrup, S. Latini, K. Bolotin, K.S. Thygesen. Phys. Rev. B 94, 041401 (2016).

[53] M. Massicotte, F. Vialla, P. Schmidt, M.B. Lundeberg, S. Latini, S. Haastrup, M. Danovich, D. Davydovskaya, K. Watanabe, T. Taniguchi, V.I. Fal'ko, K.S. Thygesen, T.G. Pedersen, F.H.L. Koppens. Nature Commun. 9, 1633 (2018).

[54] B. Brill, M. Heiblum. Phys. Rev. B 54, R17, 280 (1996).

Редактор Е.Ю. Флегонтова 